\title{
Fascin is involved in cancer cell invasion and is regulated by stromal factors
}

\author{
XIANGLAN ZHANG ${ }^{1,2^{*}}$, IL-HOON CHO ${ }^{3 *}$, JI HYEON PARK ${ }^{4}$, \\ MIN KYEONG LEE ${ }^{4}$ and YOUNG SUN HWANG ${ }^{4}$ \\ ${ }^{1}$ Yonsei University, College of Dentistry, Seoul 03722, Republic of Korea; \\ ${ }^{2}$ Department of Pathology, Yanbian University Hospital, Yanji, Jilin 133000, P.R. China; \\ Departments of ${ }^{3}$ Biomedical Laboratory Science, ${ }^{4}$ Dental Hygiene, \\ College of Health Science, Eulji University, Seongnam, Gyunggi-Do 13135, Republic of Korea
}

Received July 31, 2018; Accepted October 30, 2018

DOI: $10.3892 /$ or.2018.6847

\begin{abstract}
The tumor microenvironment plays an important role in cancer growth, invasion and metastasis. The stroma surrounding a tumor is known to contain a variety of factors that can increase angiogenesis, cancer growth and tumor progression. The aim of the present study was to determine the role of fascin in cancer growth and invasion and identify stromal factors involved in cancer progression. A fascin-depleted cell line (fascin ${ }^{\text {dep}}$ ) was used to observe the role of fascin in cancer invasion. Compared with wild-type Mock cells, cancer cell invasion in Matrigel-coated Transwell and three-dimensional (3D) culture system were reduced by fascin depletion. Tumor cell growth in vivo was also significantly reduced in mice injected with fascin ${ }^{\text {dep }}$ cells. Notably, fascin expression was increased during Transwell invasion with Matrigel compared to Transwell invasion without Matrigel. TGF- $\beta 1$, EGF and IL-1 $\beta$ significantly stimulated fascin expression. Such increased expression of fascin was also observed in cultured cells using conditioned media (CM) from cancer-associated fibroblasts (CAFs). However, no significant change in fascin expression was observed using CM from normal fibroblasts (NFs). Stimulated expression of fascin by Matrigel and CAFs was reduced by biological specific inhibitor of TGF- $\beta 1$, EGF and IL-1 $\beta$. Compared with wild-type Mock cells, the fascin ${ }^{\text {dep }}$
\end{abstract}

Correspondence to: Professor Young Sun Hwang, Department of Dental Hygiene, College of Health Science, Eulji University, 553 Sansung-Daero, Sujeong-gu, Seongnam, Gyunggi-Do 13135, Republic of Korea

E-mail: kiteys@eulji.ac.kr

${ }^{*}$ Contributed equally

Abbreviations: OSCC, oral squamous cell carcinoma; CM, conditioned media; NF, normal fibroblast; $\mathrm{CAF}$, cancer-associated fibroblast; MMP, matrix metalloprotease; Fascin ${ }^{\text {dep }}$, fascin-depleted cells

Key words: fascin, invasion, stromal fibroblast, Matrigel, xenograft cell line showed low RhoA and $\mathrm{NF}-\kappa \mathrm{B}$ activity, suggesting that RhoA and NF- $\kappa \mathrm{B}$ signals are involved in fascin expression. In conclusion, stromal factors are involved in cancer invasion and progression by activating intracellular signaling of cancer cells to increase fascin expression.

\section{Introduction}

Cancer invasion and the ability of malignant tumor cells to metastasize are closely related to cancer progression and prognosis (1). Invasive cancer is characterized by spreading beyond the layers of the developed tissue and growing into the surrounding healthy tissue. As demonstrated by numerous studies, highly invasive cancer cells have a systemic mechanism for increasing invasive activity (2). Cell-cell adhesion is lost and turned into a spindle shape while pericellular proteolysis occurs by the release of proteolytic enzyme in the direction of cancer migration followed by cancer invasion (3). Invadopodium is a protruding membrane that is actively formed in invasive cancer cells (4). It is rich in actin cytoskeleton and matrix metalloprotease (MMP). It increases the invasiveness of cancer. When infiltration is needed, cancer cells induce actin polymerization based on the Arp2/3 complex and N-WASP to stabilize the protruding structure by joining many molecules such as Tsk5, cortactin and integrin. When protease is secreted intensively around the protruding structure, the extracellular matrix is deeply degraded, acting as a mechanism to increase cancer cell invasion (2).

The regulation of invadopodium formation by various growth factors has been reported (2). Epidermal growth factor (EGF), transforming growth factor $\beta$ (TGF- $\beta$ ), platelet-derived growth factor (PDGF), hepatocyte growth factor (HGF) and heparin-binding EGF (HB-EGF) are known as typical inducers, although their activities differ depending on the type of cancer cells. Since the cancer microenvironment is closely involved in the regulation of invadopodium formation, understanding the cancer microenvironment is important for controlling cancer invasion and progression. During Transwell invasion experiments, we observed that Matrigel increased fascin expression. These expression changes were not observed in Transwell invasion experiments without Matrigel. Fascin 
is an actin bundling protein that stabilizes actin in invadopodia and increases the invasiveness of cancer (5). Matrigel ${ }^{\circledR}$ (Corning Costar, Inc., Corning, NY, USA) is composed of extracellular matrix proteins such as laminin, collagen IV and entactin. It is known to contain several growth factors. These results suggest that increased fascin expression during the infiltration process is controlled by the tumor microenvironment. The aim of the present study was to investigate stromal factors that could regulate fascin expression in cancer cells and to understand the cell signaling process involved.

\section{Materials and methods}

Cell lines and culture conditions. YD-10B human OSCC cells, which were derived from tongue cancer patient tissues, were obtained from the Department of Oral Pathology, College of Dentistry, Yonsei University (Seoul, Korea). Cells were grown in DMEM/F12 (3:1 ratio) medium supplemented with 10\% fetal bovine serum (FBS), $1 \times 10^{-10} \mathrm{M}$ cholera toxin, $0.4 \mathrm{mg} / \mathrm{ml}$ hydrocortisone, $5 \mu \mathrm{g} / \mathrm{ml}$ insulin, $5 \mu \mathrm{g} / \mathrm{ml}$ apo-transferrin and $2 \times 10^{-11} \mathrm{M}$ triiodothronine (T3) in a humidified atmosphere of $5 \% \mathrm{CO}_{2}$ at $37^{\circ} \mathrm{C}$. Normal gingival fibroblasts (NFs) were obtained from a patient who had wisdom teeth extracted and who did not have oral mucosal disease. Cancer-associated fibroblasts (CAFs) were obtained from a surgical specimen of a patient with oral squamous cell carcinoma (OSCC) and were selected from explanted cancer tissues in Versene solution [200 mg EDTA and $1 \mathrm{mg}$ glucose in 1 liter phosphate-buffered saline (PBS) buffer] (6). Briefly, the tissue was washed with betadine, cut into small pieces with scissors and washed three times with PBS. Fibroblasts were selected in Versene solution and characterized by immunohistochemical staining with anti-vimentin (dilution 1:100; cat. no. M0725; Dako, Carpinteria, CA, USA) and anti- $\alpha$-smooth muscle actin ( $\alpha$-SMA) (dilution 1:100; cat. no. ab5694; Abcam, Cambridge, MA, USA) antibodies. Early passages $(<9)$ of the fibroblasts were subjected to analysis. Informed consent was provided by the patients whose CAFs and NFs were used in this study and approval was granted by the Institutional Review Board of Yonsei University College of Dentistry. The isolated fibroblasts were maintained in DMEM/F12 (3:1 ratio) complete medium containing $1 \%$ penicillin/streptomycin. Dimethyl sulfoxide (DMSO) $[0.1 \%(\mathrm{v} / \mathrm{v})]$ was used in cell culture for control analysis.

Reagents. Dulbecco's modified Eagle's medium (DMEM), Ham's F-12 nutrient mixture, FBS, antibiotic-antimycotic $(100 \mathrm{X})$, PBS and $0.25 \%$ trypsin-EDTA (1X) were purchased from Gibco-BRL (Thermo Fisher Scientific, Inc., Waltham, MA, USA). Cholera toxin, hydrocortisone, insulin, apo-transferrin, T3 and DMSO were obtained from Sigma-Aldrich (Merck KGaA, Darmstadt, Germany). Lysophosphatidic acid (LPA) was purchased from Enzo Life Sciences (Plymouth Meeting, PA, USA). Recombinant human transforming growth factor (TGF- $\beta 1$ ), epidermal growth factor (EGF), and interleukin (IL)-1 $\beta$ were obtained from EMD Millipore (Billerica, MA, USA). SB431542, AG1478 and IL-1R antagonist were purchased from Calbiochem (Merck KGaA). The following antibodies were purchased from their respective sources: fascin (cat. no. M3567) and
E-cadherin (dilution 1:1,000; cat. no. M0725) (Dako); total (cat. no. 9242)/phosphor (cat. no. 9246) forms of IкB $\alpha$ (dilution 1:1,000; both from Cell Signaling Technology, Danvers, MA, USA); p50 (cat. no. sc-166588) and p65 (cat. no. sc-71675)

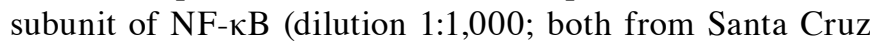
Biotechnology, Santa Cruz, CA, USA); $\beta$-actin (cat. no. A5441) and GAPDH (cat. no. G9545) (dilution 1:1,000; both from Sigma-Aldrich; Merck KGaA). Alexa Fluor 568 phalloidin and Oregon Green 488 gelatin were purchased from Molecular Probes (Thermo Fisher Scientific, Inc.).

Fascin depletion. Fascin 1-specific shRNA (h) lentiviral particles were transduced in cultured cells with $5 \mu \mathrm{g} / \mathrm{ml}$ Polybrene according to the manufacturer's protocol (Santa Cruz Biotechnology). Continual selection was followed with $1 \mathrm{mg} / \mathrm{ml}$ puromycin to establish the fascin-depleted stable cell line. Control shRNA (h) lentiviral particles-A (Santa Cruz Biotechnology) were also used as a negative control. The extent of fascin depletion was evaluated by western blot analysis.

Western blotting. Protein $(50 \mu \mathrm{g})$ was separated on $10 \%$ SDS-polyacrylamide gel and were transferred to a polyvinylidene difluoride (PVDF) membrane (EMD Millipore). The membrane was blocked with $10 \%$ skim milk in PBS containing $0.1 \%$ Tween-20 (PBS-T) and subsequently incubated overnight with a 1:1,000 dilution of the primary antibody against its specific protein at $4^{\circ} \mathrm{C}$. The blots were then incubated with a 1:3,000 dilution of their respective horseradish peroxidase-conjugated secondary antibodies for $2 \mathrm{~h}$ at room temperature and were washed with PBS-T. The targeted proteins were visualized using an enhanced chemiluminescence detection kit (Amersham Life Science, Arlington Heights, IL, USA) according to the manufacturer's instructions. Nuclear extracts were prepared using a Nonidet P-40 lysis method. Band intensities were measured with the ImageJ 1.52a software program (National Institutes of Health, Bethesda, MD, USA)

Conditioned media (CM). NFs and CAFs $\left(5 \times 10^{4}\right.$ cells) in a 100 -mm Petri dish were cultured in $6 \mathrm{ml}$ of $1 \%$ FBS media for $48 \mathrm{~h}$. The media were centrifuged at $10,000 \mathrm{x} \mathrm{g}$ for $5 \mathrm{~min}$ and the supernatant was used as CM.

Gelatin zymography. The conditioned medium was collected, and the protein concentration was determined by the Bradford method (Bio-Rad Laboratories, Hercules, CA, USA). Equal amounts of protein $(20 \mu \mathrm{g})$ were used for analysis on $8 \%$ sodium dodecyl sulfate (SDS)-polyacrylamide gel containing $0.1 \%(w / v)$ gelatin. The gel was washed with zymogram renaturation buffer (Bio-Rad Laboratories) for $1 \mathrm{~h}$ and incubated with zymogram development buffer (Bio-Rad Laboratories) for $16 \mathrm{~h}$ at $37^{\circ} \mathrm{C}$ in a shaking incubator. The gel was stained with $0.2 \%$ Coomassie Brilliant Blue and gelatinolytic activity of MMP-9 and MMP-2 were detected as clear bands in a dark blue background (7).

Invasion assay. Polycarbonate nucleopore filter inserts $(8-\mu \mathrm{m}$ pore-sized) in a 24-well Transwell chamber (Corning Costar, Cambridge, MA, USA) were coated with Matrigel (30 $\mu \mathrm{g} / \mathrm{well}$; BD Biosciences, Franklin Lakes, NJ, USA). Cells (5x10 ${ }^{4}$ cells) 
were added into the upper chamber, and complete medium was added to the bottom chamber and maintained for $48 \mathrm{~h}$ in a $37^{\circ} \mathrm{C}$ incubator. Invaded cells on the lower surface of the membrane was fixed with ethanol and non-invasive cells were removed with a cotton swab (8). Then, cells were stained with hematoxylin. Invaded cells from five fields were counted under a fluorescence microscope (EVOS ${ }^{\mathrm{TM}}$ XL Cell Imaging System; Life Technologies; Thermo Fisher Scientific, Inc.). CM was diluted 1:1 with complete media and added to the bottom of the Transwell chamber. Specific inhibitors were also added in the bottom of Transwell chamber for inhibitor studies. For fascin expression, cells in the upper and lower chamber were collected at appropriate assay times and were analyzed.

ECM degradation assay. FITC-conjugated gelatin-coated coverslips were prepared as previously described (9). Cells ( $3 \times 10^{3}$ cells) were plated on Oregon Green 488 gelatin-coated coverslips in 12-well plates and cultured for $16 \mathrm{~h}$. Cells were fixed with $4 \%$ paraformaldehyde followed by permeabilization with $0.5 \%$ Triton X-100/PBS and stained for actin with Alexa Fluor 568 phalloidin. Areas of matrix degradation was identified by a loss of fluorescence using an EVOS FL monochrome microscope (Thermo Fisher Scientific Inc.). To quantify invadopodium-mediated ECM degradation, black and white images of gelatin degradation were analyzed using ImageJ 1.52a software (National Institutes of Health).

$3 D$ culture. The cancer cells were cultured on a dermal equivalent that was generated with a Type I-A collagen mixture (Nitta Gelatin Inc., Osaka, Japan) with eight volumes of ice-cold collagen solution, one volume of $10 \mathrm{X}$ reconstitution solution $(0.022 \mathrm{~g} / \mathrm{ml} \mathrm{NaHCO}, 0.0477 \mathrm{~g} / \mathrm{ml}$ HEPES and $0.05 \mathrm{~N}$ $\mathrm{NaOH})$ and one volume 10X DMEM. Gingiva fibroblast $\left(1.5 \times 10^{5}\right.$ cells) suspensions in culture medium were then added. This mixture was poured onto polycarbonate filter inserts (3- $\mu \mathrm{m}$ pore size, $12-\mathrm{mm}$ diameter; EMD Millipore) and placed in 6-well plates (Corning Costar). After a 24-h incubation at $37^{\circ} \mathrm{C}, 3 \mathrm{ml}$ of medium was added to the 6 -well plates. Cancer cells $\left(1 \times 10^{6}\right.$ cells $)$ from each cell line were seeded onto the dermal equivalent. After $48 \mathrm{~h}$, the media in the 6-well plates were changed, and culture media were added to the epidermal equivalent. After $48 \mathrm{~h}$, the cultures were exposed to air by removing the medium from the epithelial layer to generate an air-liquid interface microenvironment. The culture medium was then changed every 2-3 days for 2 weeks. Each culture was performed independently three times and then formalin-fixed, paraffin-embedded and histologically examined. To measure invasive areas and depth, the culture tissue was stained with hematoxylin and eosin (H\&E) (6).

Luciferase reporter assay. Transcriptional activity of $\mathrm{NF}-\kappa \mathrm{B}$ was measured by luciferase reporter assay using the pNF- $\kappa$ B-Luc reporter plasmid (Clontech Laboratories, Palo Alto, CA, USA). Mock and the fascin-depleted cell line (fascin $^{\text {dep }}$ ) at 70-80\% confluence in 6-well plate were co-transfected with $1 \mu \mathrm{g}$ of NF- $\kappa \mathrm{B}$ reporter constructs and $0.5 \mu \mathrm{g}$ $\mathrm{pSV}-\beta$-galactosidase for $8 \mathrm{~h}$ in serum- and antibiotic-free Opti-MEM (Gibco-BRL; Themo Fisher Scientific, Inc.) with Lipofectamine 2000 reagent (Invitrogen; Themo Fisher Scientific, Inc.). Luciferase and $\beta$-galactosidase activities were

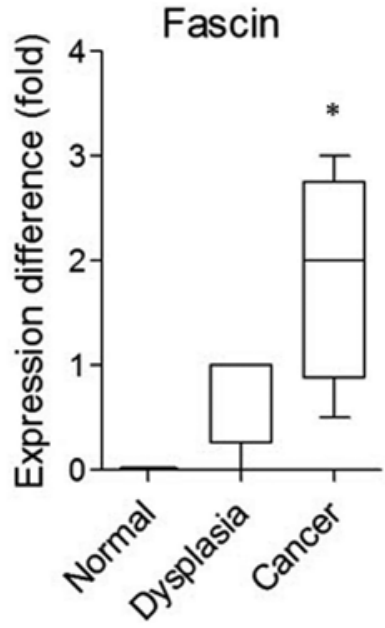

Figure 1. Differential expression of the fascin gene in normal, dysplasia and cancer tissues. Expression of fascin was analyzed in eleven oral squamous cell carcinoma (OSCC) tissues by array-comparative genomic hybridization (CGH) compared to that in normal tissues, and those with a surgical margin (dysplasia) and cancer tissues. The 60mer in situ synthesized oligonucleotide arrays containing $44 \mathrm{~K}$ probes were designed and produced by Agilent Technologies. Fluorescent dye-labeled DNA probes were prepared and hybridized onto array chips. ${ }^{*} \mathrm{P}<0.001$ vs. normal tissues.

assayed according to the manufacturer's protocol (Promega, Madison, WI, USA), using a microplate spectrofluorometer (Molecular Devices, Palo Alto, CA, USA). Luciferase activity was normalized by $\beta$-galactosidase activity in cell lysate and expressed as an average of three independent experiments. pTAL construct was used as negative control.

Animal study. All animal studies were performed in accordance with the experimental protocols that were approved by the Animal Ethics Committee of Eulji University. Male $\mathrm{Balb} / \mathrm{C}$ athymic nude mice (5 weeks of age, $10 \mathrm{~g}$ of body weight; provided by the Central Animal Laboratory, Seoul, Korea) were maintained at $20-22^{\circ} \mathrm{C}$ on a 12 -h light/dark cycle. Mock $(n=8)$ and fascin-depleted cells $\left(\right.$ fascin $\left.^{\text {dep }}\right)(n=8)$ $\left(5 \times 10^{4}\right.$ cells $/ 0.1 \mathrm{ml}$ PBS) were submucosally injected into the tongues of mice under anesthesia using a $0.5 \mathrm{ml}$ insulin syringe ( $n=5 /$ group). Growth of the tumor xenografts in mice was observed for 5 weeks. Tissues were collected and fixed in formalin and processed for paraffin embedding for histopathological studies. The tumor areas in each tongue were acquired with a fluorescence microscope (EVOS ${ }^{\mathrm{TM}}$ XL Cell Imaging system; Life Technologies; Thermo Fisher Scientific, Inc.) with a x40 magnification and digitally quantified using ImageJ 1.52a software (National Institutes of Health).

Statistical analysis. The analysis for statistical purposes was conducted using InStat GraphPad Prism version 5.01 statistical software (GraphPad Software, Inc., San Diego, CA, USA). One-way ANOVA and Tukey's honestly significant difference (HSD) post-hoc test were applied. The non-parametric Mann-Whitney test was used to evaluate the western blot analysis. Results are expressed as mean \pm standard deviation (SD). A P-value $<0.05$ was considered to indicate a statistically significant result. 
A

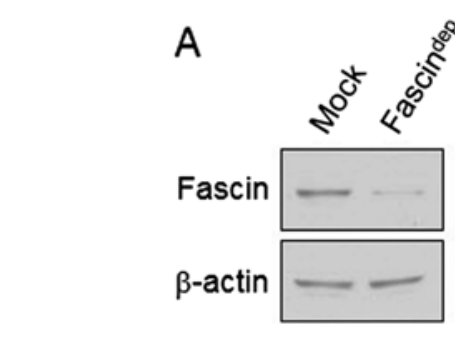

B
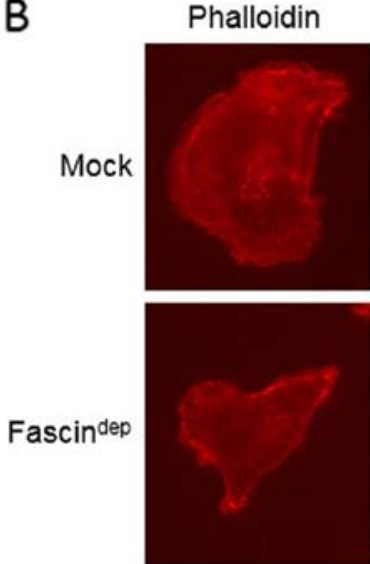

FITC-gelatin
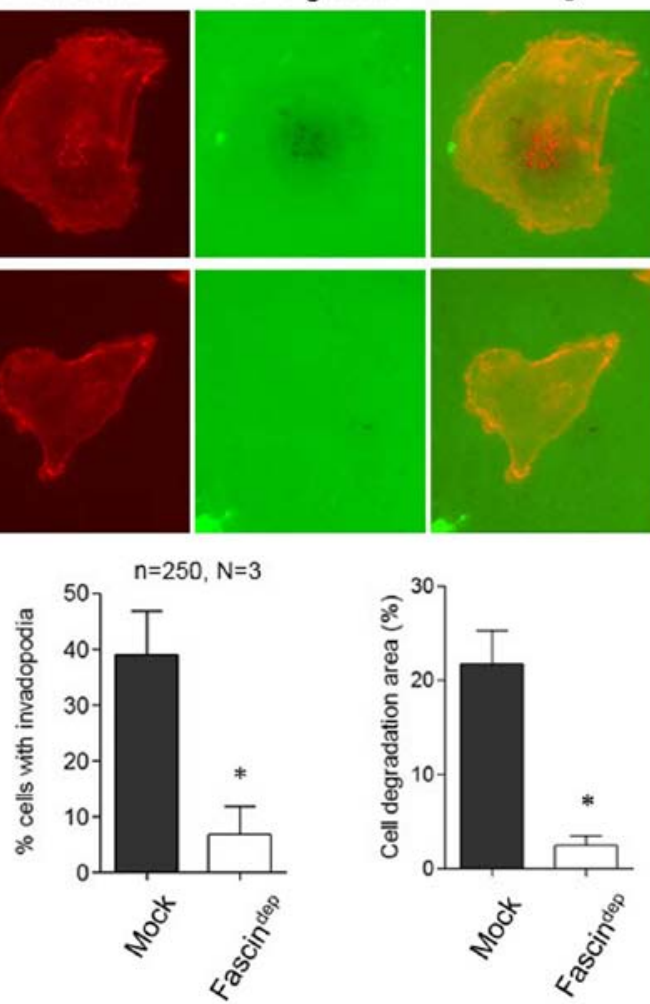

D
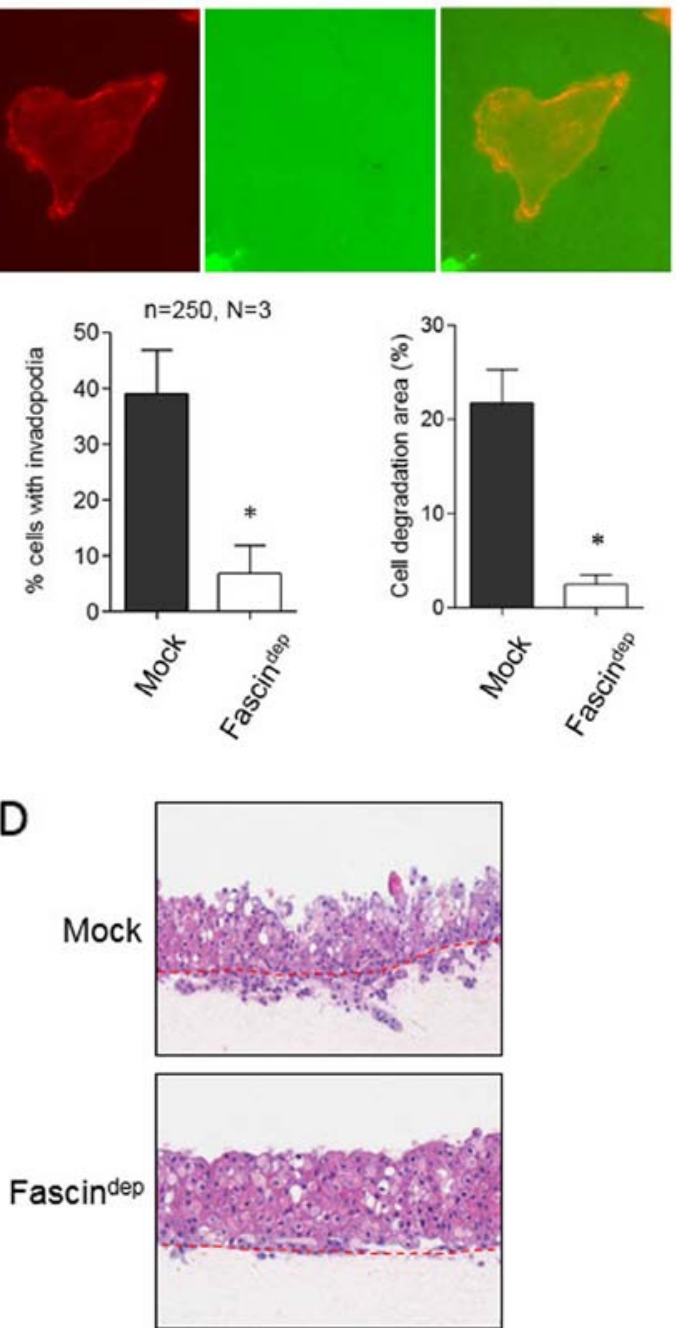

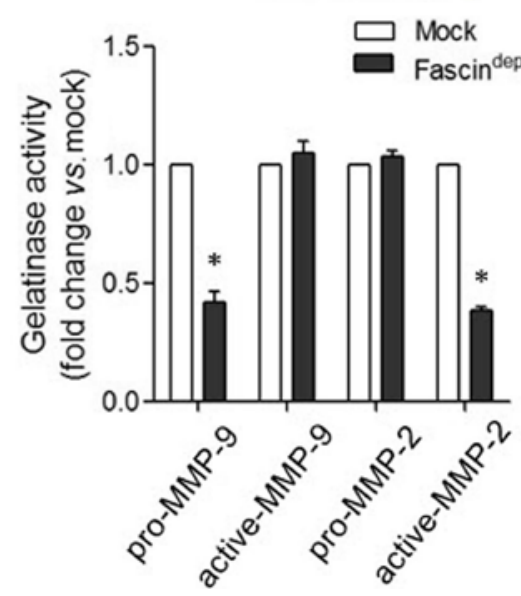

pro-MMP-9 active-MMP-9

pro-MMP-2 active-MMP-2
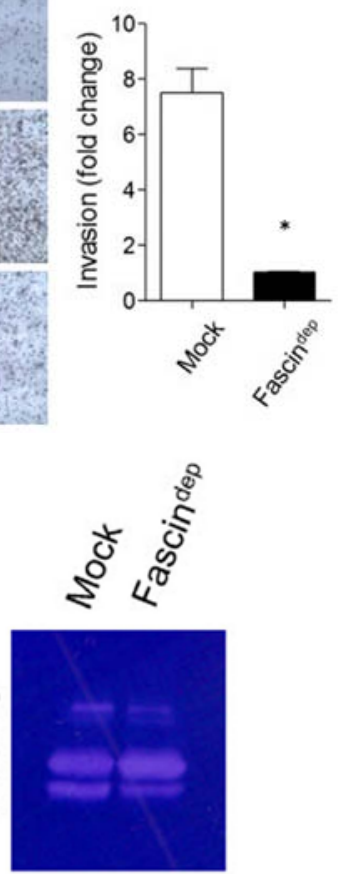

E

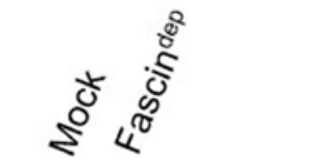


A
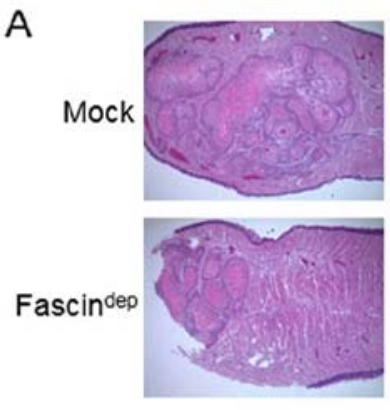
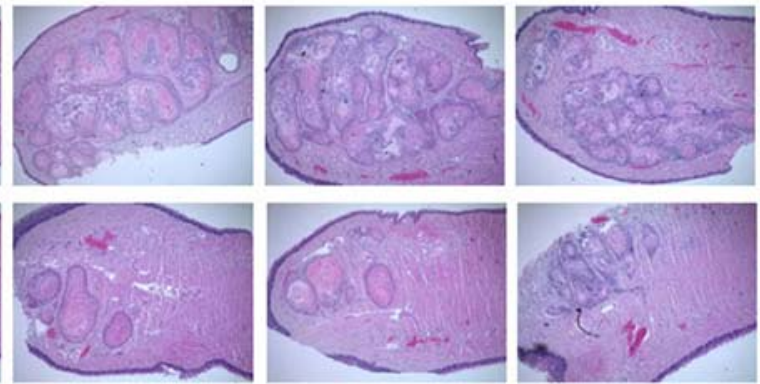
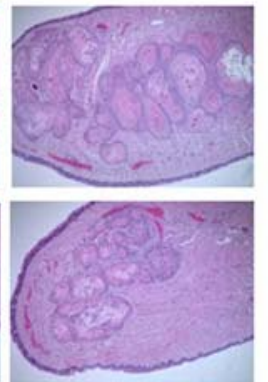

B

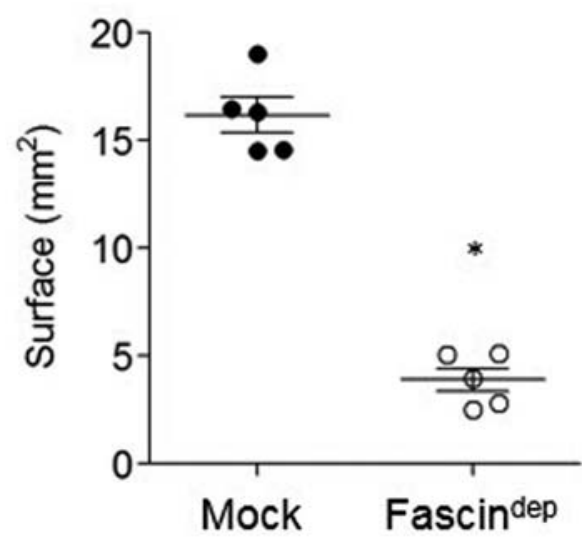

C

Mock
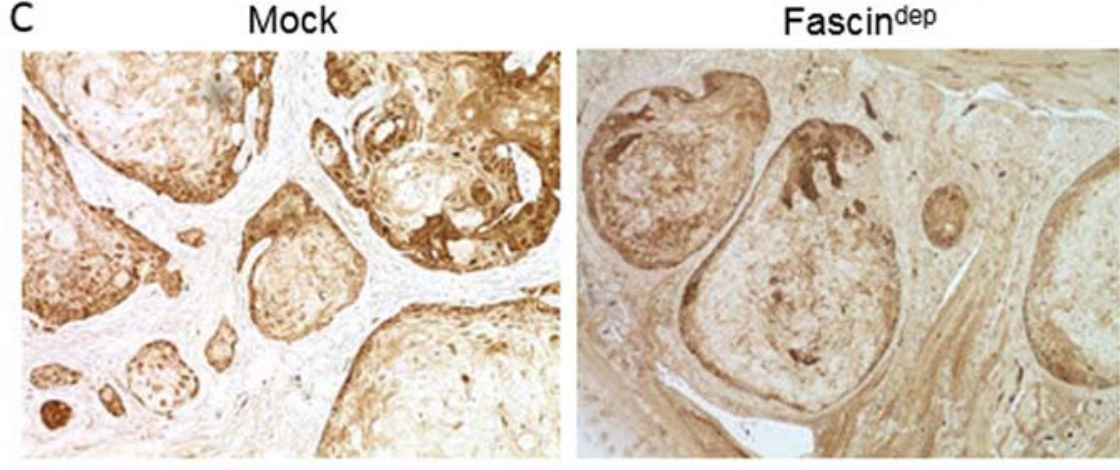

Figure 3. Effect of fascin depletion on cancer growth in mice. (A) YD-10B Mock or fascin-depleted (fascin ${ }^{\text {dep}) ~ c e l l s ~}\left(5 \times 10^{5}\right.$ cells/100 $\mu 1$ media) were submucosally injected into the tongues of mice and the growth of the tumor xenograft was observed for 5 weeks. The tumor area (in mm ${ }^{2}$ ) of each tongue in the hematoxylin and eosin (H\&E)-stained slides was acquired with a microscope with x40 magnification and (B) digitally quantified. * $<<0.001$ vs. Mock. (C) Tumor sections from the tumor xenografts were immunostained with an antibody against fascin (magnification Mock, $\mathrm{x} 20$; fascin ${ }^{\text {dep }}$, $\mathrm{x} 40$ ).

expression level in cancer specimens than that in normal specimens and dysplasia (Fig. 1). To confirm the role of fascin in OSCC, fascin depletion was performed with lentiviral short hairpin RNA (shRNA) against fascin mRNA and a stable cell line $\left(\right.$ fascin $^{\text {dep }}$ ) was established (Fig. 2A). The fascin ${ }^{\text {dep }}$ cell line had reduced invadopodia spot formation and FITC-gelatin degradation activity (Fig. 2B). This result indicates that fascin is involved in invadopodium formation and extracelluar matrix degradation activity. In the Matrigel-coated Transwell system, fascin ${ }^{\text {dep }}$ cells showed reduced invasion activity compared to wild-type Mock cells (Fig. 2C), respectively. Invasive areas into the dermal equivalents were also determined using three-dimensional (3D) cultures. Fascin ${ }^{\text {dep }}$ cells possessed low invasion activity compared to the Mock (Fig. 2D). In the zymography assay, gelatinolytic activities of pro-MMP-9 and active-MMP-2 in conditioned media (CM) from fascin ${ }^{\text {dep }}$ cells were significantly lower than those in the Mock (Fig. 2E). No statistically significant changes were observed in the active-MMP-9 and pro-MMP-2 activity.

Fascin depletion affects tumor growth in mouse xenografts. The effect of fascin on tumor growth was tested in nude mice with orthotopic tongue tumors. Submucosally inoculated Mock and fascin ${ }^{\text {dep }}$ cells were subjected to growth for 5 weeks. As shown in Fig. 3A, tumor growth was significantly increased in mice inoculated with Mock cells. However, the tumor area $\left(\mathrm{mm}^{2}\right)$ was significantly lower in the mice inoculation with the fascin ${ }^{\text {dep }}$ cells. The difference in tumor area between the two groups was statistically significant (Fig. 3B). Fascin expression was strongly observed in the invasive tumor front in the tissue of the Mock group. In the fascin ${ }^{\text {dep }}$ group, fascin expression was relatively low and encapsulated tumor was observed (Fig. 3C). 
A
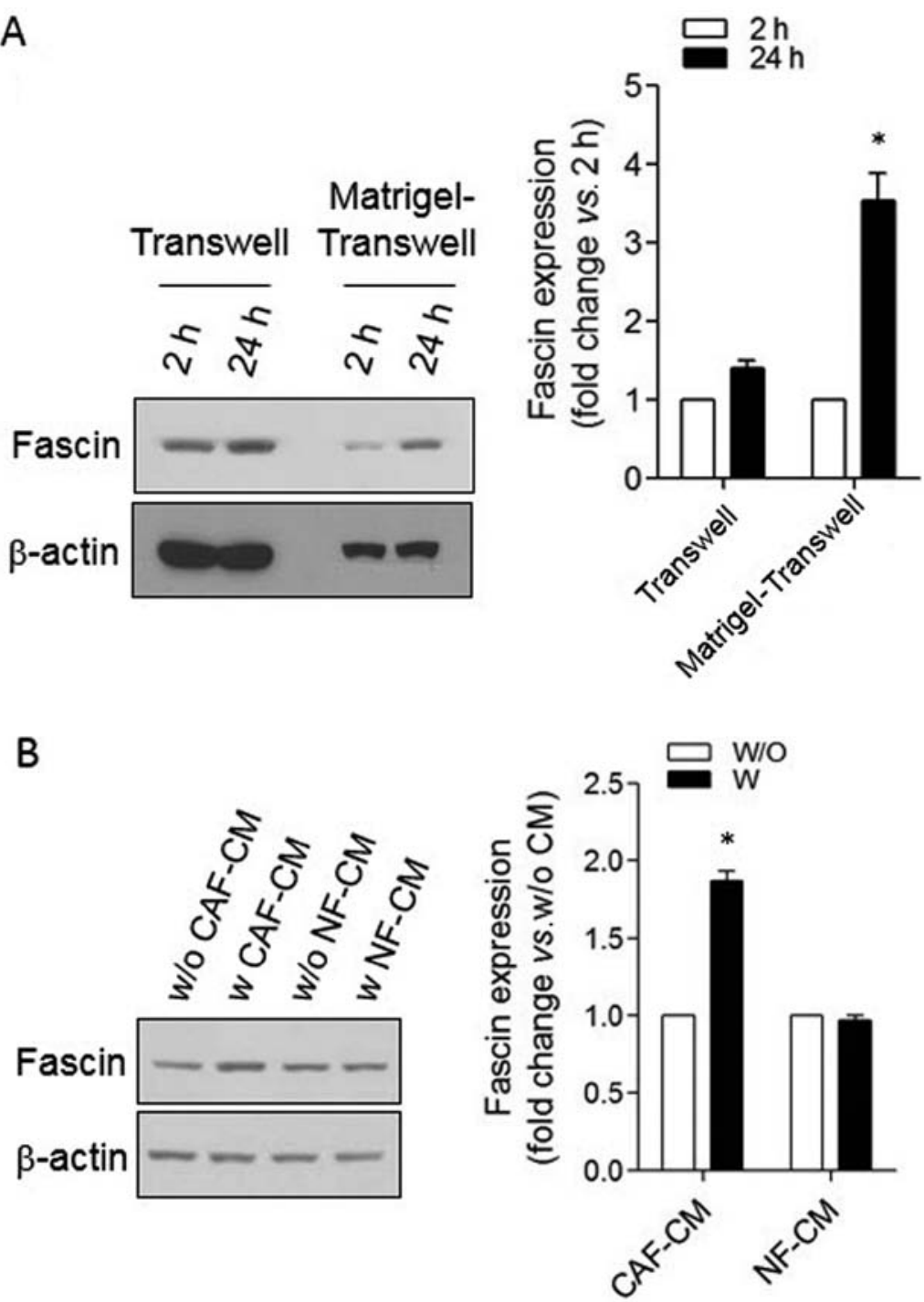

Figure 4. Analysis of regulatory factors for fascin expression. (A) Transwell invasion assay for fascin expression. Invasion assays were performed in Transwell chambers with or without Matrigel. Cells were harvested at the indicated time and western blot analysis was carried out. Each lane was selected for densitometry using the ImageJ software program and fascin expression was presented as fold change. ${ }^{*} \mathrm{P}<0.01 \mathrm{vs} .2 \mathrm{~h}$ reaction in Matrigel-coated Transwell invasion. (B) Effect of culture media (CM) from normal fibroblasts (NFs) and cancer-associated fibroblasts (CAFs) on fascin expression. Cells were cultured in 50\% diluted CM with $1 \%$ FBS media for $8 \mathrm{~h}$ and western blot analysis was performed. Band intensities were measured with ImageJ software program and fascin expression was presented as fold change. ${ }^{*} \mathrm{P}<0.05$ vs. without CAF-CM.

Fascin expression is regulated by stromal factors. To verify changes in fascin expression during the infiltration process, a Matrigel-coated Transwell invasion assay was performed. In Transwell infiltration without Matrigel, there was no significant change in fascin expression. However, expression of fascin was increased during Matrigel-coated Transwell infiltration (Fig. 4A). In addition, unlike the reaction induced by conditioned media (CM) from normal fibroblasts (NFs), the expression of fascin was significantly increased by $\mathrm{CM}$ from cancer-derived fibroblasts (CAFs) (Fig. 4B). These results indicate that the expression of fascin which occurs during invasion is regulated by the microenvironment surrounding the cancer. In order to investigate the mechanism involved in fascin expression, various molecules derived from the tumor microenvironment were used to treat cancer cells and fascin expression was examined (Fig. 4C). Lysophosphatidic acid (LPA), a phospholipid derivative that acts as a signaling molecule, was not involved in fascin expression. However, stimulation by TGF- $\beta 1$, EGF, or IL-1 $\beta$ significantly increased fascin expression. Selective inhibitor SB431542 (TGF- $\beta$ RI kinase inhibitor), AG1478 (EGF receptor tyrosine kinase inhibitor), or IL-1 receptor (IL-1R) antagonist inhibited Matrigel-induced-fascin expression (Fig. 4D). CAF stimulated-fascin expression was also diminished by treatment with SB431542, AG1478, or IL-1 receptor antagonist (Fig. 4E).

RhoA and $N F-\kappa B$ signaling are involved in fascin expression. The RhoA-NF- $\kappa B$ signaling axis plays a pivotal role in cancer invasion and motility (11). RhoA, a small GTPase protein in the Rho family, affects the invasive behavior of tumor cells by stimulating actin polymerization at the leading edge (12). Constitutively active RhoA activates NF- $\kappa \mathrm{B}$ activity during invasion (13). The YD-10B OSCC cell line used in this study is a highly invasive cancer cell line with originally very low 
C
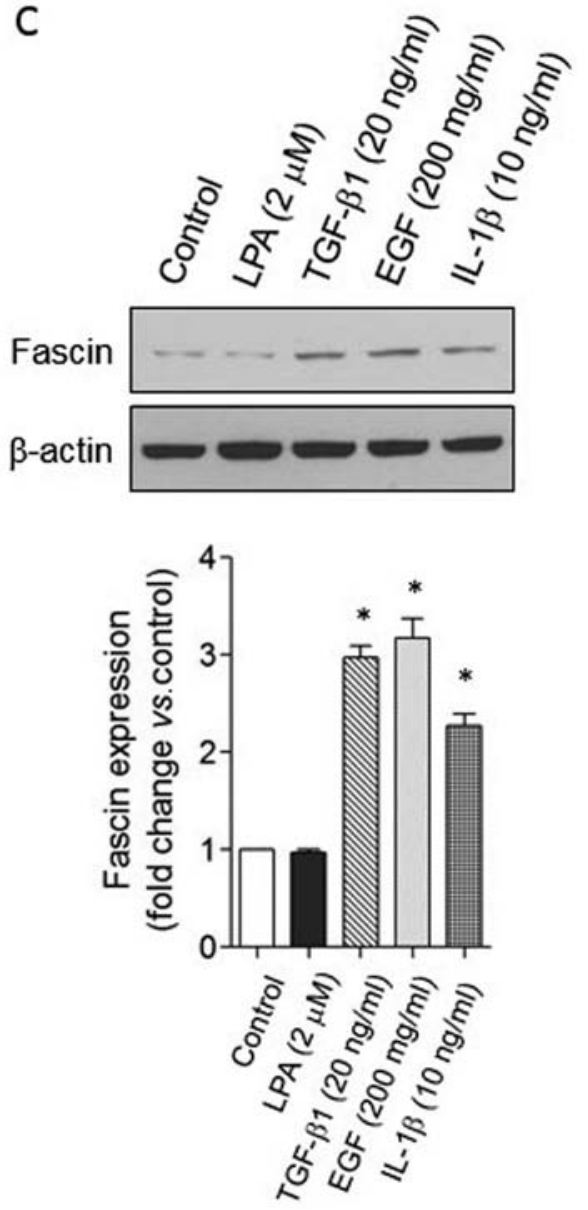

E

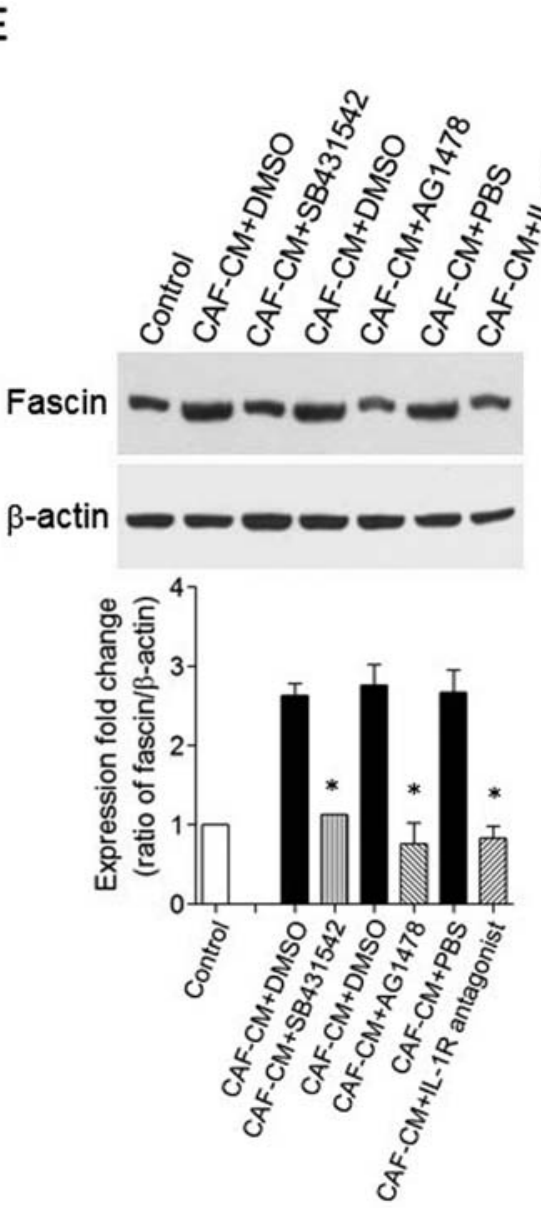

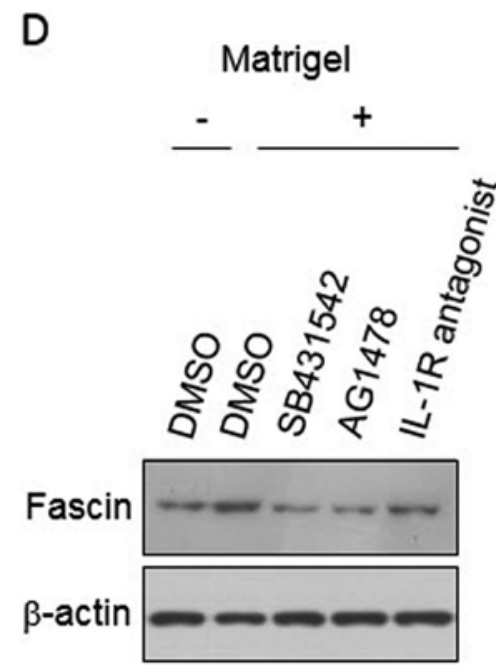

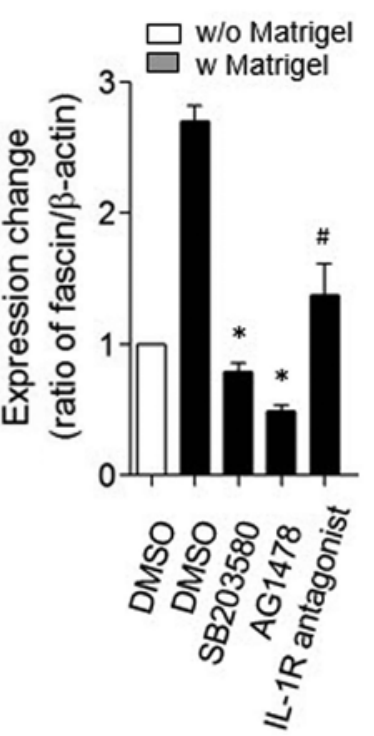

Figure 4. Continued. (C) Effect of various stimulations on fascin expression. Lysophosphatidic acid (LPA), recombinant human transforming growth factor (TGF)- $\beta 1$, epidermal growth factor (EGF), and interleukin (IL)- $1 \beta$ were used to treat the cells for $8 \mathrm{~h}$ and western blot analysis was followed. "P<0.001 vs. control. (D) Effect of biological specific inhibitors on Matrigel-stimulated fascin expression. Cells were pretreated with SB431542, AG1478, or IL-1R antagonist for $2 \mathrm{~h}$ and loaded into a Matrigel-coated Transwell chamber. Cells were harvested after $24 \mathrm{~h}$ and invasion assay and western blot analysis was carried out. Band intensities were measured with the ImageJ software program. ${ }^{~} \mathrm{P}<0.05,{ }^{*} \mathrm{P}<0.001$ vs. DMSO control in Matrigel-coated Transwell invasion. (E) Effect of biological specific inhibitors on CAF-CM-stimulated fascin expression. Cells were pretreated with SB431542, AG1478, or IL-1R antagonist for $2 \mathrm{~h}$ and cultured in 50\% diluted CM with $1 \%$ FBS media for $8 \mathrm{~h}$. Western blot analysis was performed and band intensities were measured. " $\mathrm{P}<0.05 \mathrm{vs}$. DMSO control in CAF-CM culture.

E-cadherin levels (6). In a stable cell line with dominant-negative RhoA N19, fascin expression was relatively suppressed compared to that in the Mock while the expression of epithelial phenotype marker E-cadherin was increased (Fig. 5A). 
A

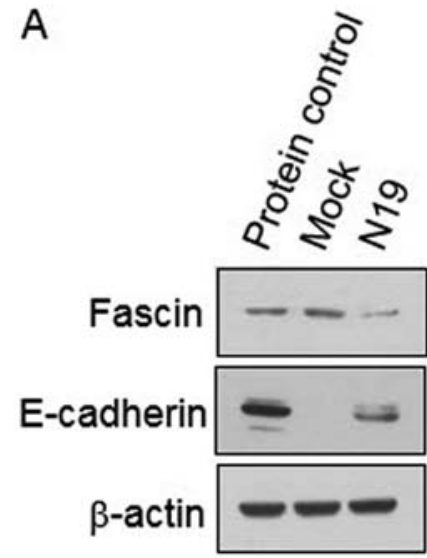

C

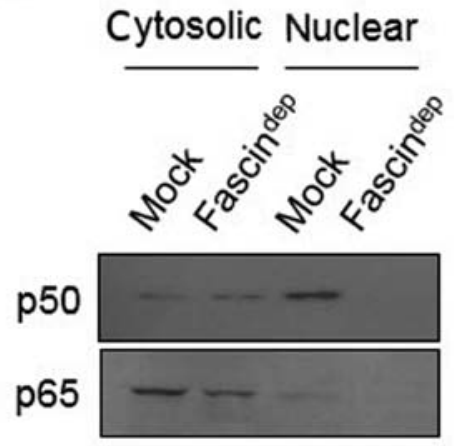

B
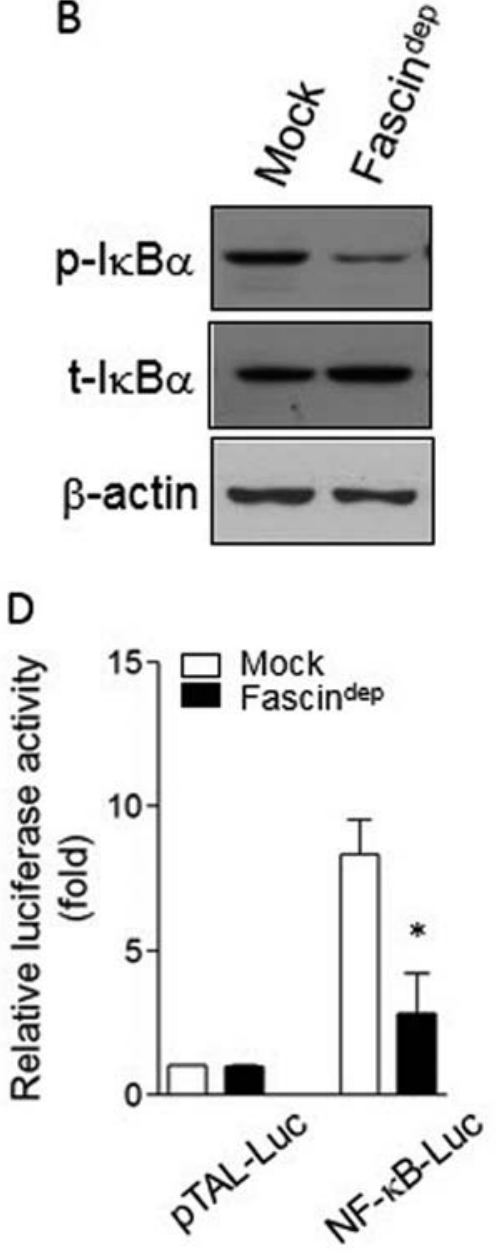

Figure 5. Analysis of signaling molecules for fascin expression. (A) Role of RhoA in fascin expression. Fascin expression was detected in dominant-negative

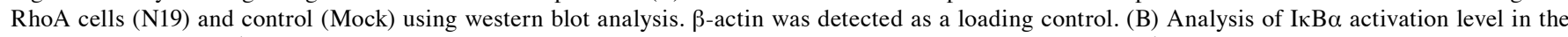
fascin-depleted (fascin ${ }^{\text {dep}}$ ) cells. Phosphorylated (p)- and total (t)-I $\kappa \mathrm{B} \alpha$ levels were monitored in the Mock and fascin ${ }^{\text {dep }}$ cells. $(\mathrm{C})$ Analysis of the NF- $\kappa \mathrm{B}$ subunit level in cytosolic and nuclear fractions from the fascin ${ }^{\text {dep }}$ cells. p50 and p65 NF- $\kappa \mathrm{B}$ subunits were analyzed in cytosolic and nuclear fractions from Mock and fascin $^{\text {dep }}$ cells by western blot analysis. (D) Analysis of NF- $\kappa$ B activation level in Mock and fascin ${ }^{\text {dep }}$ cells. Cells were transiently transfected with pTAL-Luc or p-NF- $\kappa \mathrm{B}-\mathrm{Luc}$ reporter plasmid and cultured for $24 \mathrm{~h}$. Luciferase activities were monitored and normalized to $\beta$-galactosidase after co-transfection of pRSV $\beta$-galactosidas. Data are presented as mean \pm SE from three independent experiments. ${ }^{*} \mathrm{P}<0.01$ vs. $\mathrm{p}-\mathrm{NF}-\kappa \mathrm{B}-\mathrm{Luc}$ of Mock.

In addition, I $\kappa \mathrm{B} \alpha$ an inhibitory molecule of $\mathrm{NF}-\kappa \mathrm{B}$ showed low phosphorylation levels in the fascin ${ }^{\text {dep }}$ cells (Fig. 5B). Consistent with these results, nuclear translocation levels of the p50 and p65 NF- $\mathrm{kB}$ subunit were decreased in the fascin ${ }^{\text {dep }}$ cell line compared to those in the Mock (Fig. 5C). Relatively low activity of $\mathrm{NF}-\kappa \mathrm{B}$ was found in the fascin ${ }^{\text {dep }}$ cells using a NF- $\mathrm{BB}$ luciferase reporter (Fig. 5D). These results indicate that RhoA and $\mathrm{I} \kappa \mathrm{B} \alpha / \mathrm{NF}-\kappa \mathrm{B}$ signaling are involved in fascin expression in OSCC cells.

\section{Discussion}

Stroma is a part of tissues or organs with a structural and connective role. It is composed of basement membrane, fibroblasts, extracellular matrix, immune cells and vascular system. Normal stromal tissue maintains homeostasis through its action to inhibit inflammation and cancer formation. However, it promotes cancer growth and malignancy in the tumor environment (13). Cancer cells produce several factors such as transforming growth factor (TGF)- $\beta$ that can induce transdifferentiation of normal fibroblasts to carcinoma-associated fibroblasts (CAFs), thereby serving as pivotal inducers of tumor growth, invasion and metastasis $(14,15)$. CAFs also secrete various chemokines and cytokines that can affect tumor and other stromal cells, leading to cancer growth and invasion. In addition, these factors can stimulate angiogenesis around the tumor and draw bone marrow-derived cells or immune cells around the tumor $(16,17)$. MMPs as extracellular matrix (ECM) degrading enzymes are also partly derived from CAFs (18). Therefore, the reciprocal cross-talk between stromal tissue and the tumor plays a pivotal role in cancer growth and progression.

Array-comparative genomic hybridization $(\mathrm{CGH})$ can be used to screen repeated changes of gene fragments in the genome or to observe quantitative changes in the number of repetitions. Unlike normal cells, cancer cells have structural abnormalities or chromosomal micro-deletions, thus array- $\mathrm{CGH}$ analysis is used to identify chromosomal abnormalities in cancer cells (19). In previous array-CGH studies, fascin showed a higher expression level in cancer specimens than that in normal specimens and dysplasia (10). Fascin has been reported to be involved in cancer invasion 
and has been proposed as a diagnostic marker for several tumors including breast, prostate and esophageal squamous cell carcinoma (20-25). Nevertheless, understanding of mechanisms underlying the significance of fascin expression in cancer cells is unknown, and further studies are needed. In the present study, we observed the role of fascin in cancer cell invasion using a fascin-depleted cell line and confirmed the expression mechanism of fascin by tumor stromal factors, thereby demonstrating increased cancer invasion through stroma-cancer crosstalk. YD-10 OSCC cells showed high invasive activity in a Matrigel-coated Transwell chamber system. In this experiment, infiltrated cells through the Transwell membrane were harvested and electrophoresed and increased fascin expression was observed compared to that in cells before they were placed in the Transwell chamber. According to the manufacturer's product information, Matrigel contains heparan sulfate proteoglycan (perlecan), TGF- $\beta$, epidermal growth factor (EGF), insulin-like growth factor (IGF), fibroblast growth factor (FGF), tissue plasminogen activator (tPA), and other growth factors as well as extracellular matrix proteins, such as laminin, collagen IV and entactin (https://www.corning.com/media/worldwide/cls/documents/CLS-DL-CC-026\%20DL.pdf). The increased expression of fascin induced by Matrigel during invasion indicates that fascin expression might be regulated by stimulation of stromal factors during the invasion process. To verify this possibility, normal gingival fibroblasts (NFs) and carcinoma-associated fibroblasts (CAFs) were isolated and fascin expression induced by the conditioned media from NFs and CAFs was determined. The experimental results showed that fascin expression in cancer cells was significantly increased in the culture with CAFs compared to that in the culture with NFs.

Treatment of cancer cells with growth factors resulted in increased expression of fascin in this study. It was observed that the fascin expression level was significantly increased by TGF- $\beta 1$, EGF, or IL- $1 \beta$. The increased expression of fascin was inhibited by specific inhibitors of these growth factors. Increased expression of fascin by TGF- $\beta 1$ has also been reported in gastric cancer as a Smad3 phosphorylation-dependent response (26). In addition, fascin expression by TGF- $\beta 1$ Smad4 signal was abrogated through inhibition of DNA binding of Smad4 by transcription factor GATA3 in breast cancer cells (27). We observed that fascin had a low expression level in a dominant-negative RhoA (N19) cell line. In addition, the fascin-depleted cell line (fascin ${ }^{\mathrm{dep}}$ ) showed a low phosphorylation level of $\mathrm{I} \kappa \mathrm{B} \alpha$ and translocation of NF- $\kappa \mathrm{B}$ subunit into

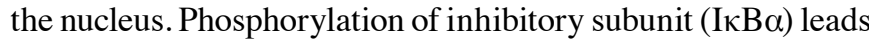
to translocation of NF- $\mathrm{KB}$ to the nucleus (11). Al-Tweigeri et al reported that fascin is a key regulator of breast cancer invasion and NF- $\mathrm{\kappa B}$ can enhance its activity (28). Fascin-deleted cells had low NF- $\mathrm{KB}$ luciferase reporter activity after TNF- $\alpha$ stimulation whereas fascin overexpressed cells showed significantly increased NF- $\mathrm{BB}$ luciferase activity after TNF- $\alpha$ stimulation. Moreover, in fascin-positive colon carcinoma cells, cAMP response element-binding protein (CREB) and aryl hydrocarbon receptor (AhR) were associated with the FSCN1 promoter region $(-219 /+114)$ and involved in the regulation of fascin transcription (29). These results indicate that fascin expression can be controlled through multiple stimuli and intracellular signaling pathways during the invasion process. RhoA and I $\kappa \mathrm{B} \alpha / \mathrm{NF}-\kappa \mathrm{B}$ signal are also involved as intracellular signals in fascin expression.

In order to understand cellular communication in vivo, it is important to mimic an in vivo cellular environment in vitro. Three-dimensional (3D) collagen matrix method is widely used to reproduce the biological environment in vitro (30). Various cellular changes such as cell invasion, differentiation, survival, and growth can be examined with this method. In the persent study, we also observed the influence of fascin depletion on invasion activity of cancer cells using 3D culture. Unlike the wild-type Mock in which the cell mass deeply invaded into the matrix, the fascin ${ }^{\text {dep }}$ cells showed low invasion activity and limited infiltration with only a few single cells. We also found that fascin was important for tumor growth through a xenograft model in mice using the fascin ${ }^{\text {dep }}$ cell line.

In conclusion, through stimulation with CAFs and growth factors, we demonstrated that fascin expression was regulated by stromal factors of the microenvironment surrounding the tumor. Gene depletion studies showed that fascin is a critical factor for cancer growth and invasion. Therefore, more research is needed to understand the reciprocal crosstalk of cancer-stroma as the cancer microenvironment is an important target for understanding cancer biology.

\section{Acknowledgements}

Not applicable.

\section{Funding}

The present study was supported by the Basic Science Research Program through the National Research Foundation of Korea (NRF) funded by the Ministry of Education, Science and Technology (nos. 2015R1D1A1A01056946 and 2018R1D1A1B07042035).

\section{Availability of data and materials}

The datasets used during the present study are available from the corresponding author upon reasonable request.

\section{Authors' contributions}

YSH, XZ and IHC designed the research, analyzed the data, wrote and revised the manuscript. YSH, XZ, IHC, JHP and MKL performed the experiments. All authors read and approved the manuscript and agree to be accountable for all aspects of the research in ensuring that the accuracy or integrity of any part of the work are appropriately investigated and resolved.

\section{Ethics approval and consent to participate}

All studies were performed in accordance with experimental protocols that were approved by the Animal Ethics Committee of Eulji University and by the Institutional Review Board of Yonsei University College of Dentistry. The samples were used according to ethical standards. 


\section{Patient consent for publication}

Not applicable.

\section{Competing interests}

The authors state that they have no competing interests.

\section{References}

1. Guan X: Cancer metastases: Challenges and opportunities. Acta Pharm Sin B 5: 402-418. 2015.

2. Eddy RJ, Weidmann MD, Sharma VP and Condeelis JS: Tumor cell invadopodia: Invasive protrusions that orchestrate metastasis. Trends Cell Biol 27: 595-607, 2017.

3. Brabletz T, Kalluri R, Nieto MA and Weinberg RA: EMT in cancer. Nat Rev Cancer 18: 128-134, 2018.

4. Gould CM and Courtneidge SA: Regulation of invadopodia by the tumor microenvironment. Cell Adh Migr 8: 226-235, 2014

5. Li A, Dawson JC, Forero-Vargas M, Spence HJ, Yu X, König I, Anderson K and Machesky LM: The actin-bundling protein fascin stabilizes actin in invadopodia and potentiates protrusive invasion. Curr Biol 20: 339-345, 2010

6. Kim EJ, Che ZM, Park YJ, Hwang YS, Kim KY, Jung DW Jeon NK, Choi YW, Lee EJ and Kim J: Morphogenesis and biological significance of spindle cell transformation in a spindle cell carcinoma. Cancer Lett 275: 61-71, 2009.

7. Toth M, Sohail A and Fridman R: Assessment of gelatinases (MMP-2 and MMP-9) by gelatin zymography. Methods Mol Biol 878: 121-135, 2012.

8. Hwang YS, Park KK and Chung WY: Stromal transforming growth factor-beta 1 is crucial for reinforcing the invasive potential of low invasive cancer. Arch Oral Biol 59: 687-694, 2014.

9. Bowden ET, Coopman PJ and Mueller SC: Invadopodia: Unique methods for measurement of extracellular matrix degradation in vitro. Methods Cell Biol 63: 613-627, 2001.

10. Hwang YS, Park KK, Cha IH, Kim J and Chung WY: Role of insulin-like growth factor-II mRNA-binding protein-3 in invadopodia formation and the growth of oral squamous cell carcinoma in athymic nude mice. Head Neck 34: 1329-1339, 2012.

11. Hodge JC, Bub J, Kaul S, Kajdacsy-Balla A and Lindholm PF: Requirement of RhoA activity for increased nuclear factor kappaB activity and PC-3 human prostate cancer cell invasion. Cancer Res 63: 1359-1364, 2003.

12. Struckhoff AP, Rana MK and Worthylake RA: RhoA can lead the way in tumor cell invasion and metastasis. Front Biosci (Landmark Ed) 16: 1915-1926, 2011

13. Bremnes RM, Dønnem T, Al-Saad S, Al-Shibli K, Andersen S, Sirera R, Camps C, Marinez I and Busund LT: The role of tumor stroma in cancer progression and prognosis: Emphasis on carcinoma-associated fibroblasts and non-small cell lung cancer. J Thorac Oncol 6: 209-217, 2011.

14. Shiga $K$, Hara M, Nagasaki $T$, Sato $T$, Takahashi $H$ and Takeyama H: Cancer-associated fibroblasts: Their characteristics and their roles in tumor growth. Cancers 7: 2443-2458, 2015.

15. De Wever O and Mareel M: Role of tissue stroma in cancer cell invasion. J Pathol 200: 429-447, 2003.
16. Li J, Jia Z, Kong J, Zhang F, Fang S, Li X, Li W, Yang X, Luo Y, Lin B, et al: Carcinoma-associated fibroblasts lead the invasion of salivary gland adenoid cystic carcinoma cells by creating an invasive track. PLoS One 11: e0150247, 2016.

17. Wen S, Niu Y, Yeh S and Chang C: BM-MSCs promote prostate cancer progression via the conversion of normal fibroblasts to cancer-associated fibroblasts. Int J Oncol 47: 719-727, 2015.

18. Taniwaki K, Fukamachi H, Komori K, Ohtake Y, Nonaka T, Sakamoto T, Shiomi T, Okada Y, Itoh T, Itohara S, et al: Stroma-derived matrix metalloproteinase (MMP)-2 promotes membrane type 1-MMP-dependent tumor growth in mice. Cancer Res 67: 4311-4319, 2007.

19. van Beers EH and Nederlof PM: Array-CGH and breast cancer. Breast Cancer Res 8: 210, 2006.

20. Al-Alwan M, Olabi S, Ghebeh H, Barhoush E, Tulbah A, Al-Tweigeri T, Ajarim D and Adra C: Fascin is a key regulator of breast cancer invasion that acts via the modification of metastasis-associated molecules. PLoS One 6: e27339, 2011.

21. Huang FK, Han S, Xing B, Huang J, Liu B, Bordeleau F, Reinhart-King CA, Zhang JJ and Huang XY: Targeted inhibition of fascin function blocks tumour invasion and metastatic colonization. Nat Commun 6: 7465, 2015.

22. Min KW, Chae SW, Kim DH, Do SI, Kim K, Lee HJ, Sohn JH, Pyo JS, Kim DH, Oh SJ, et al: Fascin expression predicts an aggressive clinical course in patients with advanced breast cancer. Oncol Lett 10: 121-130, 2015.

23. Darnel AD, Behmoaram E, Vollmer RT, Corcos J, Bijian K, Sircar K, Su J, Jiao J, Alaoui-Jamali MA and Bismar TA: Fascin regulates prostate cancer cell invasion and is associated with metastasis and biochemical failure in prostate cancer. Clin Cancer Res 15: 1376-1383, 2009.

24. Wang CQ, Tang CH, Chang HT, Li XN, Zhao YM, Su CM, Hu GN, Zhang T, Sun XX, Zeng Y, et al: Fascin-1 as a novel diagnostic marker of triple-negative breast cancer. Cancer Med 5: 1983-1988, 2016.

25. Takikita M, Hu N, Shou JZ, Giffen C, Wang QH, Wang C, Hewitt SM and Taylor PR: Fascin and CK4 as biomarkers for esophageal squamous cell carcinoma. Anticancer Res 31: 945-952, 2011.

26. Li L, Cao F, Liu B, Luo X, Ma X and Hu Z: TGF- $\beta$ induces fascin expression in gastric cancer via phosphorylation of smad3 linker area. Am J Cancer Res 5: 1890-1896, 2015.

27. Sun J, He H, Pillai S, Xiong Y, Challa S, Xu L, Chellappan S and Yang S: GATA3 transcription factor abrogates Smad4 transcription factor-mediated fascin overexpression, invadopodium formation, and breast cancer cell invasion. J Biol Chem 288: 36971-36982, 2013.

28. Ghebeh H, Al-Khaldi S, Olabi S, Al-Dhfyan A, Al-Mohanna F, Barnawi R, Tulbah A, Al-Tweigeri T, Ajarim D and Al-Alwan M: Fascin is involved in the chemotherapeutic resistance of breast cancer cells predominantly via the PI3K/Akt pathway. Br J Cancer 111: 1552-1561, 2014.

29. Hashimoto Y, Loftis DW and Adams JC: Fascin-1 promoter activity is regulated by CREB and the aryl hydrocarbon receptor in human carcinoma cells. PLoS One 4: e5130, 2009.

30. Langhans SA: Three-dimensional in vitro cell culture models in drug discovery and drug repositioning. Front Pharmacol 9: 6, 2018. 